\title{
An Analytical Solution of Rayleigh Wave Propagation Problem on Elastic Substrate with Nonconductive Viscoelastic Layer
}

\section{PASTERNAK*}

Institute of Radioelectronics, Military University of Technology, S. Kaliskiego 2, 00-490 Warsaw, Poland

The Rayleigh wave propagation problem in the elastic substrate-viscoelastic layer interface was analysed in the paper. The fundamental solution of the problem has been found in the Fourier-Laplace space using Biot viscoelastic solid model with Maxwell relaxation time. The space-time form of the solution was obtained using Cagniard-deHoop method. The solution presented in the paper seems to be applicable to surface acoustic waves gas sensors with polymeric (viscoelastic) layer.

PACS numbers: 43.35.Pt, 46.35.+z, 68.35.Ja

\section{Introduction}

The problem of the Rayleigh wave propagation on substrates with nonconductive viscoelastic (polymeric) layers is very important if the surface acoustic wave (SAW) sensors technology development is considered. Such layers are often used for complex organic vapours detection. As they have viscoelastic properties, the behaviour of whole SAW gas detector is determined by elastic substrate and viscoelastic layer as well as their interface parameters.

Although some approximate solutions of the problem had already been found (e.g. [1-7]), yet the general solution remained unknown. The fundamental solution is interesting because it contains important information about the parameters of SAW sensor with viscoelastic (polymeric) chemisensitive layer. The properties of elastic substrate and viscoelastic layer (geometrical and material) as well as optimal value of the sensor operational frequency are of primordial importance.

\section{Solution of the problem}

The equation system formulated in [3] fulfils the following boundary conditions that assume vanishing of the stresses at the top of the layer $\left(\sigma_{13}^{\mathbb{1}}=0, \sigma_{33}^{\mathbb{1}}=0\right.$ for $\left.x_{3}=0\right)$ and equality of respective displacements and stresses at the interface $\left(u_{1}^{(1)}=u_{1}^{(2)}, u_{3}^{(1)}=u_{3}^{(2)}, \sigma_{13}^{(1)}=\sigma_{13}^{(2)}\right.$, $\sigma_{33}^{(1)}=\sigma_{33}^{(2)}$ for $\left.x_{3}=h\right)$. In the Fourier-Laplace transforms space it has the form as follows:

$$
\begin{aligned}
& \hat{\bar{\sigma}}_{13}^{(1)}=2 \mathrm{i} \alpha \bar{\gamma}_{1}^{(1)} \bar{\mu}^{(1)}\left(-\bar{A}^{(1)}+\bar{B}^{(1)} \mathrm{e}^{-2 \bar{\gamma}_{1}^{(1)} h}\right)-\bar{\mu}^{(1)} \\
& \quad \times\left\{\left[\alpha^{2}-\left(\bar{\gamma}_{2}^{(1)}\right)^{2}\right]\left(\bar{C}_{2}^{(1)}+\bar{D}_{2}^{(1)} \mathrm{e}^{-2 \bar{\gamma}_{2}^{(1)} h}\right)\right\}=0,
\end{aligned}
$$

\footnotetext{
* e-mail: MateuszPasternak@we.wat.edu.pl
}

$$
\begin{aligned}
& \hat{\bar{\sigma}}_{33}^{(1)}=-\bar{\lambda}^{(1)} \alpha^{2}\left(\bar{A}^{(1)}+\bar{B}^{(1)} \mathrm{e}^{-2 \bar{\gamma}_{1}^{(1)} h}\right) \\
& +\left(\bar{\lambda}^{(1)}+2 \bar{\mu}^{(1)}\right)\left(\bar{\gamma}_{1}^{(1)}\right)^{2}\left(\bar{A}^{(1)}+\bar{B}^{(1)} \mathrm{e}^{-h \bar{\gamma}_{1}^{(1)} h}\right) \\
& +2 \mathrm{i} \alpha \bar{\gamma}_{2}^{(\mathbb{1}} \bar{\mu}^{(\mathbb{1}}\left(-\bar{C}_{2}^{(\mathbb{1}}+D_{2}^{(\mathbb{1}} \mathrm{e}^{-2 \bar{\gamma}_{2}^{(1)} h}\right)=0, \\
& \mathrm{i} \alpha\left(\bar{A}^{(1)} \mathrm{e}^{-\bar{\gamma}_{1}^{(1)} h}+\bar{B}^{(\mathbb{1}} \mathrm{e}^{-\bar{\gamma}_{1}^{(1)} h}\right)+\bar{\gamma}_{2}^{(\mathbb{1}} \bar{C}_{2}^{(\mathbb{1})} \mathrm{e}^{-\bar{\gamma}_{2}^{(1)} h}-\bar{\gamma}_{2}^{(1)} \\
& \times \bar{D}_{2}^{(1)} \mathrm{e}^{-\bar{\gamma}_{2}^{(1)} h}=\mathrm{i} \alpha A^{(2)} \mathrm{e}^{-\gamma_{1}^{(2)} h}+\bar{\gamma}_{2}^{(2)} C_{2}^{(2)} \mathrm{e}^{-\gamma_{2}^{(2)} h}, \\
& -\bar{\gamma}_{1}^{(1)} \bar{A}^{(1)} \mathrm{e}^{-\bar{\gamma}_{1}^{(1)} h}+\bar{\gamma}_{1}^{(1)} \bar{B}^{(1)} \mathrm{e}^{-\bar{\gamma}_{1}^{(1)} h}+\mathrm{i} \alpha\left(\bar{C}_{2}^{(1)} \mathrm{e}^{-\bar{\gamma}_{2}^{(1)} h}\right. \\
& \left.+\bar{D}_{2}^{(1)} \mathrm{e}^{-\bar{\gamma}_{2}^{(1)} h}\right)=-\bar{\gamma}_{1}^{(2)} A^{(2)} \mathrm{e}^{-\gamma_{1}^{(2)} h}+\mathrm{i} \alpha C_{2}^{(2)} \mathrm{e}^{-\gamma_{2}^{(2)} h},( \\
& 2 \mathrm{i} \alpha \bar{\gamma}_{1}^{(1)} \bar{\mu}^{(1)}\left(-\bar{A}^{(1)} \mathrm{e}^{-\bar{\gamma}_{1}^{(1)} h}+\bar{B}^{(1)} \mathrm{e}^{-\bar{\gamma}_{1}^{(1)} h}\right) \\
& -\bar{\mu}^{(1)}\left\{\left[\alpha^{2}-\left(\bar{\gamma}_{2}^{(1)}\right)^{2}\right]\left(\bar{C}_{2}^{(\mathbb{1})} \mathrm{e}^{-\bar{\gamma}_{2}^{(1)} h}+\bar{D}_{2}^{(1} \mathrm{e}^{-\bar{\gamma}_{2}^{(1)} h}\right)\right\} \\
& =-2 \mathrm{i} \alpha \mu^{(2)} \gamma_{1}^{(2)} A^{(2)} \mathrm{e}^{-\gamma_{1}^{(2)} h} \\
& -\mu^{(2)}\left[\alpha^{2}+\left(\gamma_{2}^{(2)}\right)^{2}\right] C_{2}^{(2)} \mathrm{e}^{-\gamma_{2}^{(2)} h}, \\
& -\bar{\lambda}^{(1)} \alpha^{2}\left(\bar{A}^{\mathbb{1}} \mathrm{e}^{-\gamma_{1}^{(\mathbb{D}} h}+\bar{B}^{\mathbb{1}} \mathrm{e}^{-\bar{\gamma}_{1}^{(\mathbb{1}} h}\right) \\
& +\left(\bar{\lambda}^{(1)}+2 \bar{\mu}^{(\mathbb{1}}\right)\left(\bar{\gamma}_{1}^{(\mathbb{1}}\right)^{2}\left(\bar{A}^{\mathbb{1}} \mathrm{e}^{-\gamma_{1}^{(1)} h}+\bar{B}^{\mathbb{1}} \mathrm{e}^{-\bar{\gamma}_{1}^{(1)} h}\right) \\
& +2 \mathrm{i} \alpha \bar{\gamma}_{2}^{(1)} \bar{\mu}^{(1)}\left(-\bar{C}_{2}^{(\mathbb{1})} \mathrm{e}^{-\bar{\gamma}_{2}^{(1)} h}+D_{2}^{(\mathbb{1})} \mathrm{e}^{-\bar{\gamma}_{2}^{(1)} h}\right) \\
& =\left[-\lambda^{(2)} \alpha^{2}+\left(\lambda^{(2)}+2 \mu^{(2)}\right)\left(\gamma_{1}^{(2)}\right)^{2}\right] A^{(2)} \mathrm{e}^{-\gamma_{1}^{(2)} h} \\
& -2 \mathrm{i} \alpha \mu^{(2)}\left(\gamma_{2}^{(2)} C_{2}^{(2)} \mathrm{e}^{-\gamma_{2}^{(2)} h}\right)
\end{aligned}
$$


where superscripts (1) and (2) denote the quantities for the layer and the substrate, respectively, $\sigma$ - the stress tensor, $h$ - layer thickness, $\lambda$ and $\mu$ are the Lamé constants (time dependent for the viscoelastic layer),

$$
\begin{aligned}
& \bar{\gamma}_{1}^{(1)}=\sqrt{\frac{s^{2} \rho^{(1)}}{\lambda^{(1)}(s)+2 \mu^{(1)}(s)}+\alpha^{2},} \\
& \bar{\gamma}_{2}^{(1)}=\sqrt{\frac{s^{2} \rho^{(1)}}{\mu^{(1)}(s)}+\alpha^{2},} \\
& \bar{\gamma}_{1}^{(2)}=\sqrt{\frac{s^{2} \rho^{(2)}}{\lambda^{(2)}+2 \mu^{(2)}}+\alpha^{2}}, \\
& \bar{\gamma}_{2}^{(2)}=\sqrt{\frac{s^{2} \rho^{(2)}}{\mu^{(2)}}+\alpha^{2}},
\end{aligned}
$$

$\rho$ is the density, $\alpha$ and $s$ Fourier and Laplace transform arguments, respectively. The time dependence of the Lamé constants for the layer was described using the Biot model with the Maxwell relaxation time $\tau$ as follows:

$$
\begin{aligned}
& \lambda^{(1)}(t)=\lambda^{(1)} \delta(t-\tau)-\frac{\lambda^{(1)}}{\tau} \exp \left(-\frac{t}{\tau}\right), \\
& \mu^{(1)}(t)=\mu^{(1)} \delta(t-\tau)-\frac{\mu^{(\mathbb{1}}}{\tau} \exp \left(-\frac{t}{\tau}\right),
\end{aligned}
$$

where $\lambda^{(1)}$ and $\mu^{(1)}$ are time independent.

In the matrix representation the equation system has the following form:

$$
[\boldsymbol{M}]\left[A^{(1)} A^{(2)} B^{(1)} C_{2}^{(1)} C_{2}^{(2)} D_{2}^{(1)}\right]^{\mathrm{T}}=0 .
$$

The solution of the characteristic equation $\operatorname{det}[M]=0$ was presented in [3]. It describes the Rayleigh wave velocity variation as a function of substrate and the layer parameters as well as the layer thickness. The numerical tests have shown that the viscoelastic layer reduce the Rayleigh wave velocity from a fraction to even hundreds of $\mathrm{m} / \mathrm{s}$ and is linear when the layer geometrical and material parameters are changing. Such behaviour is verified experimentally.

The solution does not contain any information about frequency dependence because the Laplace transform argument $s$ has been eliminated from the characteristic equation.

In order to find the frequency dependence the following nonhomogeneous system with free stimulating stresses $N_{i}$ at the substrate-layer interface on the right-hand side was solved:

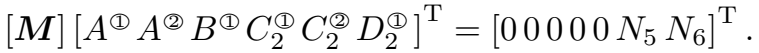

The roots of Eq. (3) were calculated using the Cramer method. Inserting them into the displacements formulae (see [3]) one can obtain the displacements at the viscoelastic layer and substrate-layer interface. The displacements have complicated algebraic form.

In order to inverse the displacements from the FourierLaplace transform space into space-time the terms dependent on $s$ were separated and remaining terms were treated as constants modifying amplitudes only. How- ever, amplitudes contain some functions depending on $s$ but it can be demonstrated that for time long enough the functions become constants and equal to

$\sqrt{\bar{\lambda}{ }^{(1)}+2 \bar{\mu}^{\circledR}}$ or $\sqrt{\bar{\mu}^{(1}}$.

Using Cagniard-deHoop method [7] the inverse Laplace transforms can be found according to the formula

$$
\begin{gathered}
\overline{\boldsymbol{u}}\left(x_{1}, x_{3}, t\right)=\boldsymbol{L}_{t}^{-1}\left\{\left.\operatorname{Res}\left[\hat{\bar{u}}\left(x_{3}, \alpha, s\right)\right]\right|_{\alpha=\alpha_{\mathrm{R}}}\right\} \\
=\boldsymbol{L}_{t}^{-1}\left\{2 \pi \mathrm{i} \lim _{\alpha \rightarrow \alpha_{\mathrm{R}}} \hat{\overline{\boldsymbol{u}}}\left(x_{3}, \alpha_{\mathrm{R}}, s\right)\left(\alpha-\alpha_{\mathrm{R}}\right)\right\},
\end{gathered}
$$

where $\alpha_{\mathrm{R}}$ is the root of characteristic equation [3]

Applying (4) the displacements take the form as follows:

$$
\begin{aligned}
\overline{\boldsymbol{u}} & =\boldsymbol{A} \int_{-\infty}^{\infty} \frac{\Phi(\alpha, s)}{W^{\prime}(\alpha, s)} \mathrm{e}^{-\bar{\gamma}_{1,2}^{(1)}(\alpha) x_{3}} \mathrm{e}^{\mathrm{i} \alpha x_{1}} \mathrm{~d} \alpha \\
& =\boldsymbol{A} \frac{\Phi\left(\alpha_{\mathrm{R}}, s\right)}{W^{\prime}\left(\alpha_{\mathrm{R}}\right)} \mathrm{e}^{-\bar{\gamma}_{1,2}^{(1)}\left(\alpha_{\mathrm{R}}\right) x_{3}} \mathrm{e}^{\mathrm{i} \alpha_{\mathrm{R}} x_{1}},
\end{aligned}
$$

where $\boldsymbol{A}$ denotes the amplitude and $\Phi$ are Cramer's rule numerators, $W^{\prime}$ is the first derivative of $\operatorname{det}[M]$.

Formula (5) allows to calculate the displacements in the form as follows:

$$
\begin{aligned}
& \bar{u}_{1}^{(1)}=\frac{1}{\boldsymbol{A} s^{2}} \mathrm{e}^{\mathrm{i} x_{\mathrm{R}} x_{1}}\left[a_{1} \mathrm{e}^{-s f_{1} x_{3} \sqrt{v_{11}^{2}+x_{\mathrm{R}}^{2}}}\right. \\
& +a_{2} \mathrm{e}^{-s f_{1}\left(2 h-x_{3}\right) \sqrt{v_{11}^{2}+x_{\mathrm{R}}^{2}}} \\
& +a_{3} \mathrm{e}^{-s f_{1}\left[\frac{h}{\beta} \sqrt{v_{21}^{2}+x_{\mathrm{R}}^{2}}+\left(h-x_{3}\right) \sqrt{v_{11}^{2}+x_{\mathrm{R}}^{2}}\right]} \\
& +a_{4} \mathrm{e}^{-s f_{2} x_{3} \sqrt{v_{21}^{2}+x_{\mathrm{R}}^{2}}} \\
& +a_{5} \mathrm{e}^{-s f_{2}\left[\left(h-x_{3}\right) \sqrt{v_{21}^{2}+x_{\mathrm{R}}^{2}}+\beta h \sqrt{v_{11}^{2}+x_{\mathrm{R}}^{2}}\right]} \\
& \left.+a_{6} \mathrm{e}^{-s f_{2}\left(2 h-x_{3}\right) \sqrt{v_{21}^{2}+x_{\mathrm{R}}^{2}}}\right] \\
& \bar{u}_{3}^{(1)}=\frac{1}{\boldsymbol{B} s^{2}} \mathrm{e}^{\mathrm{i} x_{\mathrm{R}} x_{1}}\left[b_{1} \mathrm{e}^{-s f_{1} x_{3}} \sqrt{v_{11}^{2}+x_{\mathrm{R}}^{2}}\right. \\
& +b_{2} \mathrm{e}^{-s f_{1}\left(2 h-x_{3}\right) \sqrt{v_{11}^{2}+x_{\mathrm{R}}^{2}}} \\
& +b_{6} \mathrm{e}^{\left.-s f_{1}\left(2 h-x_{3}\right) \sqrt{v_{21}^{2}+x_{\mathrm{R}}^{2}}\right]} \\
& +b_{3} \mathrm{e}^{-s f_{1}\left[\frac{h}{\beta} \sqrt{v_{21}^{2}+x_{\mathrm{R}}^{2}}+\left(h-x_{3}\right) \sqrt{v_{11}^{2}+x_{\mathrm{R}}^{2}}\right]} \\
& +b_{4} \mathrm{e}^{-s f_{2} x_{3} \sqrt{v_{21}^{2}+x_{\mathrm{R}}^{2}}} \\
& -s f_{2}\left[\left(h-x_{3}\right) \sqrt{v_{21}^{2}+x_{\mathrm{R}}^{2}}+\beta h \sqrt{v_{11}^{2}+x_{\mathrm{R}}^{2}}\right]
\end{aligned}
$$




$$
\begin{aligned}
& \bar{u}_{1}^{(2)}=\frac{1}{\boldsymbol{C} s^{2}} \mathrm{e}^{\mathrm{i} x_{\mathrm{R}} x_{1}}\left[c_{1} \mathrm{e}^{-s\left[\left(x_{3}-h\right) \sqrt{v_{12}^{2}+x_{\mathrm{R}}^{2}}+f_{1} h \sqrt{v_{11}^{2}+x_{\mathrm{R}}^{2}}\right]}\right. \\
& +c_{2} \mathrm{e}^{-s\left[\left(x_{3}-h\right) \sqrt{v_{12}^{2}+x_{\mathrm{R}}^{2}}+f_{2} h \sqrt{v_{21}^{2}+x_{\mathrm{R}}^{2}}\right]} \\
& +c_{3} \mathrm{e}^{-s\left[\left(x_{3}-h\right) \sqrt{v_{22}^{2}+x_{\mathrm{R}}^{2}}+f_{1} h \sqrt{v_{11}^{2}+x_{\mathrm{R}}^{2}}\right]} \\
& +c_{4} \mathrm{e}^{\left.-s\left[\left(x_{3}-h\right) \sqrt{v_{22}^{2}+x_{\mathrm{R}}^{2}}+f_{2} h \sqrt{v_{21}^{2}+x_{\mathrm{R}}^{2}}\right]\right]}, \\
& \bar{u}_{3}^{(2)}=\frac{1}{\boldsymbol{D} s^{2}} \mathrm{e}^{\mathrm{i} x_{\mathrm{R}} x_{1}}\left[d_{1} \mathrm{e}^{-s\left[\left(x_{3}-h\right) \sqrt{v_{12}^{2}+x^{2}}+f_{1} h \sqrt{v_{11}^{2}+x_{\mathrm{R}}^{2}}\right]}\right. \\
& +d_{2} \mathrm{e}^{-s\left[\left(x_{3}-h\right) \sqrt{v_{12}^{2}+x_{\mathrm{R}}^{2}}+f_{2} h \sqrt{v_{21}^{2}+x_{\mathrm{R}}^{2}}\right]} \\
& +d_{3} \mathrm{e}^{-s\left[\left(x_{3}-h\right) \sqrt{v_{22}^{2}+x_{\mathrm{R}}^{2}}+f_{1} h \sqrt{v_{11}^{2}+x_{\mathrm{R}}^{2}}\right]} \\
& \left.+d_{4} \mathrm{e}^{-s\left[\left(x_{3}-h\right) \sqrt{v_{22}^{2}+x_{\mathrm{R}}^{2}}+f_{2} h \sqrt{v_{21}^{2}+x_{\mathrm{R}}^{2}}\right]}\right],
\end{aligned}
$$

where

$$
f_{1}=\sqrt{\frac{s+\frac{1}{\tau}}{\bar{\mu}_{0}^{(1)} s}}, \quad f_{2}=\sqrt{\frac{s+\frac{1}{\tau}}{\left(\bar{\lambda}_{0}^{(1)}+2 \bar{\mu}_{0}^{(\mathbb{D}}\right) s}}
$$

$\beta=f_{1} / f_{2}, \boldsymbol{A}, \boldsymbol{B}, \boldsymbol{C}, \boldsymbol{D}$ and $a_{i}, b_{i}, c_{i}, d_{i}$ are constant coefficients depending on material parameters of the substrate and the layer as well as layer thickness.

Applying $\alpha_{\mathrm{R}}=s x_{\mathrm{R}}$ the formula for $\tilde{u}_{1}^{(1)}$ takes the following form:

$$
\begin{aligned}
& \tilde{u}_{1}^{(1)}=\frac{1}{\boldsymbol{A}} \int_{0}^{t}(t-\eta) H(t-\eta) a_{1} \Omega\left(x_{3} \sqrt{\frac{v_{11}^{2}+x_{\mathrm{R}}^{2}}{\lambda^{(1)}+2 \mu^{(1)}}}\right) \\
& +a_{2} \Omega\left(\left(2 h-x_{3}\right) \sqrt{\frac{v_{11}^{2}+x_{\mathrm{R}}^{2}}{\lambda^{\oplus}+2 \mu^{(1)}}}\right) \\
& +a_{3} \Omega\left(\frac{h}{\beta} \sqrt{\frac{v_{21}^{2}+x_{\mathrm{R}}^{2}}{\lambda^{(1)}+2 \mu^{(1)}}}+\left(h-x_{3}\right) \sqrt{\frac{v_{11}^{2}+x_{\mathrm{R}}^{2}}{\lambda^{(1)}+2 \mu^{(1)}}}\right) \\
& +a_{4} \Omega\left(x_{3} \sqrt{\frac{v_{21}^{2}+x_{\mathrm{R}}^{2}}{\mu^{2}}}\right) \\
& +a_{5} \Omega\left(\left(h-x_{3}\right) \sqrt{\frac{v_{21}^{2}+x_{\mathrm{R}}^{2}}{\mu^{(2)}}}+\beta h \sqrt{\frac{v_{11}^{2}+x_{\mathrm{R}}^{2}}{\mu^{(2)}}}\right) \\
& +a_{6} \Omega\left(\left(2 h-x_{3}\right) \sqrt{\frac{v_{21}^{2}+x_{\mathrm{R}}^{2}}{\mu^{(2)}}}\right) \mathrm{d} \eta,
\end{aligned}
$$

where $\lambda^{(1)}$ and $\mu^{(1)}$ denote instantaneous parts of $\bar{\lambda}^{(1)}$ and $\bar{\mu}^{(\mathbb{1}}$, respectively, $v_{11}, v_{21}, v_{12}, v_{22}$ are inverses of velocities of longitudinal and transversal waves in the layer and longitudinal and transversal waves in the substrate, respectively, $x_{\mathrm{R}}$ is interpreted as reverse of the Rayleigh wave at the interface,

$$
\begin{aligned}
& \Omega(\kappa) \equiv \mathrm{e}^{-\frac{t}{2 \tau}} \\
& \quad \times\left[\delta(t-\kappa)+\frac{\tau^{2}\left(2 t^{2}-\kappa^{2}\right)}{\pi t\left(2 \tau^{2}-\kappa^{2}\right)} \mathrm{e}^{\frac{1}{2 \tau}\left(t-\frac{\kappa^{2}}{2 t}\right)} H(t-\kappa)\right],
\end{aligned}
$$

where Bessel function asymptotic form was used. Assuming harmonic forces $N_{i}=U_{i} \mathrm{e}^{\mathrm{i} k x_{1}} \mathrm{e}^{-\chi x_{3}} \mathrm{e}^{\mathrm{i} \omega t}$, where $\chi$ is the attenuation coefficient, the displacement $u_{1}^{(1)}$ in the space-time obtains the form of convolution

$$
\begin{aligned}
u_{1}^{(1)} & =\tilde{u}_{1}^{(\mathbb{1}}(. ; t) * N_{i 0} \mathrm{e}^{\mathrm{i} \omega t} \\
& =N_{i 0} \int_{0}^{t} \mathrm{e}^{\mathrm{i} \omega(t-\eta)} \tilde{u}_{1}^{(\mathbb{1}}(. ; \eta) \mathrm{d} \eta .
\end{aligned}
$$

The integral (8) is the consequence of $s^{2}$ existing in the denominator of (6a) but the integration result changes amplitude only.

Applying (8) the displacement $u_{1}^{(1)}$ takes the following form:

$$
\begin{aligned}
u_{1}^{(1)} & =\mathrm{e}^{\mathrm{i} x_{\mathrm{R}} x_{1}} \int_{0}^{t} \mathrm{e}^{\mathrm{i} \omega(t-\eta)} \mathrm{e}^{-\frac{t}{2 \tau}} \\
& \times\left[\delta(\eta-\kappa)+\frac{\tau^{2}\left(2 \eta^{2}-\kappa^{2}\right)}{\pi \eta\left(2 \tau^{2}-\kappa^{2}\right)} \mathrm{e}^{\frac{1}{2 \tau}\left(\eta-\frac{\kappa^{2}}{2 \eta}\right)}\right] \mathrm{d} \eta \\
& =\mathrm{e}^{\mathrm{i} x_{\mathrm{R}} x_{1}} \mathrm{e}^{-\frac{t}{2 \tau}}\left[\mathrm{e}^{\mathrm{i} \omega(t-\varpi)}+\mathrm{e}^{\mathrm{j} \omega t} \int_{0}^{t} \frac{\tau^{2}\left(2 \eta^{2}-\kappa^{2}\right)}{\pi \eta\left(2 \tau^{2}-\kappa^{2}\right)}\right. \\
& \left.\times \mathrm{e}^{\frac{1}{2 \tau}\left(\eta-\frac{\kappa^{2}}{2 \eta}\right)} \mathrm{e}^{-\mathrm{i} \omega \eta} \mathrm{d} \eta\right]=\mathrm{e}^{\mathrm{i} x_{\mathrm{R}} x_{1}} \mathrm{e}^{-\frac{t}{2 \tau}}\left\{\mathrm{e}^{\mathrm{i} \omega(t-\varpi)}\right. \\
& +\mathrm{e}^{\mathrm{j} \omega t}\left[\int_{0}^{\infty} \frac{\tau^{2}\left(2 \eta^{2}-\kappa^{2}\right)}{\pi \eta\left(2 \tau^{2}-\kappa^{2}\right)} \mathrm{e}^{\frac{1}{2 \tau}\left(\eta-\frac{\kappa^{2}}{2 \eta}\right)} \mathrm{e}^{-\mathrm{i} \omega \eta} \mathrm{d} \eta+\right. \\
& \left.\left.-\int_{t}^{\infty} \frac{\tau^{2}\left(2 \eta^{2}-\kappa^{2}\right)}{\pi \eta\left(2 \tau^{2}-\kappa^{2}\right)} \mathrm{e}^{\frac{1}{2 \tau}\left(\eta-\frac{\kappa^{2}}{2 \eta}\right)} \mathrm{e}^{-\mathrm{i} \omega \eta} \mathrm{d} \eta\right]\right\}
\end{aligned}
$$

where $\mathrm{e}^{\mathrm{i} \omega(t-\varpi)}=\sum_{m=1}^{4} \mathrm{e}^{\mathrm{i} \omega\left(t-\zeta_{m}\right)}$,

$$
\begin{aligned}
& \zeta_{1}=x_{3}\left[\sqrt{\frac{v_{11}^{2}+x_{\mathrm{R}}^{2}}{\lambda^{(1)}+2 \mu^{(1)}}}+\sqrt{\frac{v_{21}^{2}+x_{\mathrm{R}}^{2}}{\mu^{(2)}}}\right] \text {, } \\
& \zeta_{2}=\left(2 h-x_{3}\right)\left[\sqrt{\frac{v_{11}^{2}+x_{\mathrm{R}}^{2}}{\lambda^{(1)}+2 \mu^{\perp}}}+\sqrt{\frac{v_{21}^{2}+x_{\mathrm{R}}^{2}}{\mu^{(2)}}}\right] \text {, } \\
& \zeta_{3}=\left(h-x_{3}\right)\left[\sqrt{\frac{v_{11}^{2}+x_{\mathrm{R}}^{2}}{\lambda^{(1)}+2 \mu^{(1)}}}+\sqrt{\frac{v_{21}^{2}+x_{\mathrm{R}}^{2}}{\mu^{(2)}}}\right] \text {, } \\
& \zeta_{4}=\frac{h}{\beta} \sqrt{\frac{v_{21}^{2}+x_{\mathrm{R}}^{2}}{\lambda^{(1)}+2 \mu^{(1)}}}+\beta h \sqrt{\frac{v_{11}^{2}+x_{\mathrm{R}}^{2}}{\mu^{(2)}}} .
\end{aligned}
$$

It can be shown that in such a representation the Heaviside functions inside $\Omega(\kappa)$ are equal to 1 . The last integral term in (9) vanishes in the stationary state. The displacements $u_{1}^{(2)} u_{2}^{(1)}$ and $u_{2}^{(2)}$ can be calculated in the 
same way. Finally, in the stationary state all displacements obtain the similar general form

$$
u_{j}^{(\mathcal{D}}=\Psi_{j} \mathrm{e}^{\mathrm{i} \Phi\left(t, x_{1}\right)},
$$

where $\Psi_{j}$ are the general amplitudes different for individual displacements and $\Phi$ - general phase the same for all displacements. It has the following form:

$$
\begin{aligned}
& \Phi\left(; x_{1}, t\right)=\ln \left(\exp \left(\mathrm{i} \omega x_{1} x_{\mathrm{R}}-\frac{t}{2 \tau}\right)\right. \\
& \times \exp \left(2 \exp \left(i \omega\left(t-h \sqrt{\frac{\frac{\rho_{1}}{\lambda_{1}+2 \mu_{1}}+x_{\mathrm{R}}^{2}}{\lambda_{1}+2 \mu_{1}}}\right)\right)\right. \\
& +\exp \left(i \omega\left(t-h \sqrt{\mu_{1} \frac{v_{21}^{2}+x_{\mathrm{R}}^{2}}{\left(\lambda_{1}+2 \mu_{1}\right)^{2}}}\right)\right) \\
& +2 \exp \left(\mathrm{i} \omega\left(t-h \sqrt{\frac{v_{21}^{2}+x_{\mathrm{R}}^{2}}{\mu_{2}}}\right)\right) \\
& +2 \exp \left(i \omega\left(t-h \sqrt{\frac{\lambda_{1}+2 \mu_{1}}{\mu_{1}}} \sqrt{\frac{\frac{\rho_{1}}{\lambda_{1}+2 \mu_{1}}+x_{\mathrm{R}}^{2}}{\mu_{2}}}\right)\right) \text {. }
\end{aligned}
$$

The general form of angular frequency and wave number can be calculated by the well known formulae

$$
\tilde{\omega}=\frac{\mathrm{d} \Phi}{\mathrm{d} t}, \quad \tilde{k}=\frac{\mathrm{d} \Phi}{\mathrm{d} x_{1}},
$$

and the general forms of phase and group velocities of the Rayleigh wave

$$
\tilde{v}_{\mathrm{p}}=\frac{\tilde{k}}{\tilde{\omega}}, \quad \tilde{v}_{\mathrm{g}}=\frac{\mathrm{d} \tilde{k}}{\mathrm{~d} \tilde{\omega}} .
$$

Applying (12) and (13) one can obtain the inverse velocities in the following form:

$$
\begin{aligned}
& \tilde{v}_{\mathrm{p}}=\frac{2 \omega \tau x_{\mathrm{R}}}{\mathrm{i}+2 \omega \tau}=\frac{4 \omega^{2} \tau^{2} x_{\mathrm{R}}}{4 \omega^{2} \tau^{2}+1}-\mathrm{i} \frac{2 \omega \tau x_{\mathrm{R}}}{4 \omega^{2} \tau^{2}+1}, \\
& \tilde{v}_{\mathrm{g}}=x_{\mathrm{R}} .
\end{aligned}
$$

The result is similar to the well known relation for viscoelastic media but instead of viscoelastic complex moduli uses the root of dispersion relation.
The inversed imaginary part of is $\tilde{v}_{\mathrm{p}}$ responsible for wave attenuation and real one describes the velocity change versus frequency and relaxation time of viscoelastic layer. When $\omega \tau \gg 0.5$ the relation between phase velocity and frequency vanishes and phase velocity becomes equal to group velocity that is equal to the root of dispersion relation.

\section{Conclusion}

The approach presented here is flexible enough to calculate the response of the elastic substrate-viscoelastic layer system for free elastic stimulations at the top of the system or interface. Actually, to analyse the Rayleigh wave velocity changes at the system interface for large $\omega \tau$ it is enough to calculate the root of dispersion equation (the way to calculate the root has been described in [3]). It is dependent on the material parameters of the substrate and the layer as well as the layer thickness and is approximately independent of frequency. The results allow to conclude that increase the SAW sensor operational frequency for maximise its response is valuable when the condition $\omega \tau \gg 0.5$ is fulfilled. Practically, it means that the frequency increase gives positive effect for the layers with short relaxation times only.

\section{References}

[1] A. Auld, Acoustic Fields and Waves in Solids, Wiley Interscience, New York 1973.

[2] S.J. Martin, G.C. Frye, S.D. Senturia, Anal. Chem. 66, 2201 (1994).

[3] M. Pasternak, Acta Phys. Pol. A 114, 167 (2008).

[4] T. Pustelny, B. Pustelny, Europ. Phys. J. - Special Topics 154, 265 (2008).

[5] T. Pustelny, A. Opilski, B. Pustelny, Acta Phys. Pol. A 114, A-181 (2008).

[6] W. Jakubik, M. Urbanczyk, E. Maciak, T. Pustelny, Bull. Pol. Acad. Sci. 56, 133 (2008).

[7] A.T. deHoop, Handbook of Radiation and Scattering of Waves. Acoustic Waves in Fluids, Elastic Waves in Solids, Electromagnetic Waves, Academic Press, 1995. 